\title{
General Results of Existence and Asymptotic Stability for a class of Electromechanical Systems
}

\author{
Márcio José Horta Dantas \\ Faculdade de Matemática, UFU, \\ 38400-902 Uberlândia M.G. \\ E-mail: marcio@ufu.br \\ Rubens Sampaio \\ Roberta Lima \\ Mechanical Engineering Department, PUC-Rio \\ Rua Marquês de São Vicente, 225; 38097, Rio de Janeiro \\ E-mail:rsampaio@puc-rio.br roberta_10_lima@hotmail.com.
}

\begin{abstract}
In this work a class of time-dependent electromechanical system is investigated. From general results on existence and stability of periodic orbits, the dynamics of this system can be approached in a mathematically rigorous way. These results generalize previous ones obtained for autonomous electromechanical systems.
\end{abstract}

Key words: Electromechanical System, Periodic Orbits, Stability, Nonlinear Dynamics 1 Introduction

Nowadays the electromechanical coupling is a common fact in our technology. Moreover, the dynamics of electromechanical systems is a topic that is worthy to research due to not only for its importance in applications, but because it leads to very interesting dynamical systems whose investigation is interesting by itself $[2,6,7,8,9]$. Motors are the most used actuator in technology.

In this paper a theoretical analysis is performed in a very simple system composed by a cart, fixed elastically to a wall and with viscous dissipation, and whose motion is driven by a DC motor. The coupling between the motor and the cart is made by a mechanism called scotch yoke. In this simple system the coupling is a sort of master-slave condition: the motor drives, the cart is driven, and that is all.

This paper is organized as follows. In Section 2 the equations of motion are deduced from first principles. In Section 3 the equations are rewritten in a dimensionless form and an Ansatz is made for the derivation of the main results of the paper. Included in the Ansatz is the careful selection of parameters that is crucial for the simplification of the computations. In Section 4 three theorems are stated without proof due to space limitation. One of them, the Theorem 4.3, establishes a result of stability which gives exactly what is necessary to show the asymptotic stability of the angular speed and the current of the electromechanical system under investigation. Then, in Section 5 these theorems are applied to our system. It is interesting to say that this approach generalizes our results given in [1], because now one can deal with time-dependent electromechanical systems whereas the earlier approach is inadequate for them. It is worth to note these results were motivated by the numerical research of [7]. The main conclusions are presented in Section 6. 


\section{An Electromechanical System}

The mathematical modeling of DC motors is based on the Kirchhoff's law [5]. It is constituted by the equations

$$
\begin{aligned}
& l c^{\prime}(t)+r c(t)+k_{e} \alpha^{\prime}(t)=\nu+\chi \sin \left(\omega_{1} t\right), \\
& j_{m} \alpha^{\prime \prime}(t)+b_{m} \alpha^{\prime}(t)-k_{t} c(t)=-\tau(t),
\end{aligned}
$$

where $t$ is the time, $\nu$ and $\chi$ are constant voltages, $c$ is the electric current, $\dot{\alpha}$ is the angular speed of the motor, $l$ is the electric inductance, $j_{m}$ is the motor moment of inertia, $b_{m}$ is the damping ratio in the transmission of the torque generated by the motor to drive the coupled mechanical system, $k_{t}$ is the torque constant, $k_{e}$ is the motor electromagnetic force constant and $r$ is the electrical resistance. Figure 1 shows a sketch of a DC motor. The available torque to the coupled mechanical system is represented by $\tau$, that is the component of the torque vector $\vec{\tau}$ in the $z$ direction shown in Figure 1 .

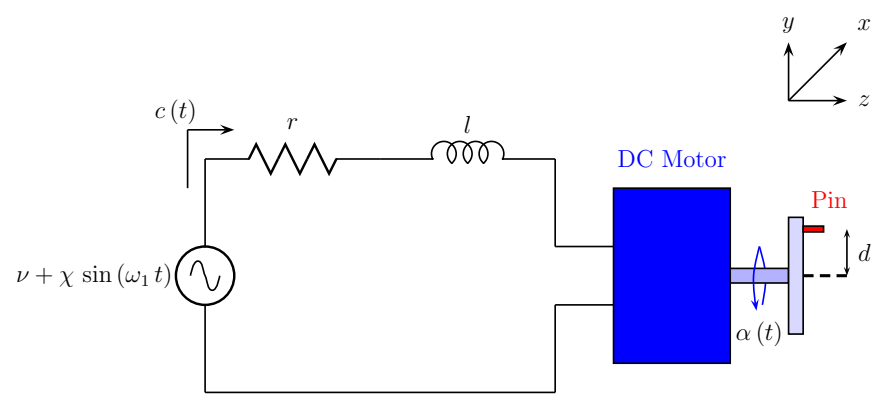

Figure 1: Eletrical DC Motor

The system analyzed in this paper is composed by a cart fixed elastically to a wall with viscous dissipation and whose motion is driven by the DC motor as sketched in Figure 2. The motor is coupled to the cart through a pin that slides into a slot machined on an acrylic plate that is part to the cart, as shown in Figure 2. The pin hole is drilled off-center on a disk fixed in the axis of the motor, so that the motor rotational motion is transformed into horizontal cart motion. On this cart is applied an elastic force $-c_{1} x$ and a viscous damping given by $-k_{1} x^{\prime}$.

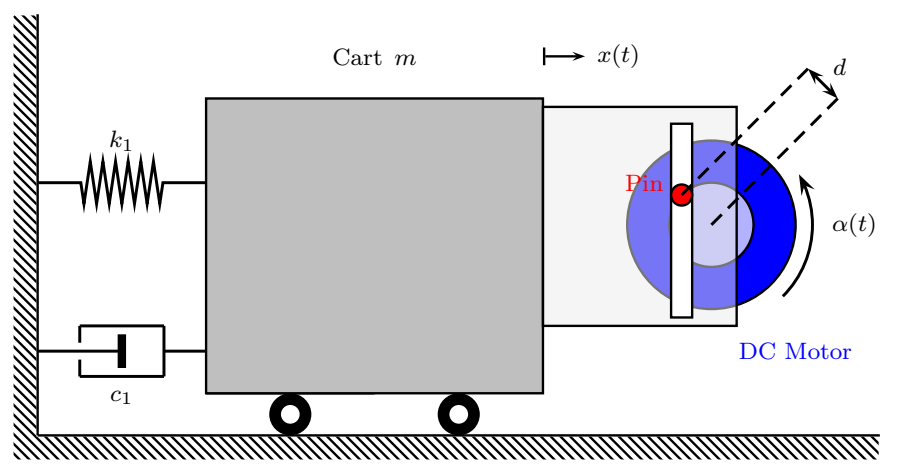

Figure 2: Electromechanical System

To model the coupling between the motor and the mechanical system, it is assumed that the motor shaft is rigid. Thus, the available torque to the coupled mechanical system, $\vec{\tau}$, can be written as 


$$
\vec{\tau}(t)=\vec{d}(t) \times \vec{f}(t),
$$

where $\vec{d}$ is the eccentricity of the pin of the motor and $\vec{f}$ is the coupling force between the DC motor and the cart. By the problem geometry, the module of $\vec{d}$ is the nominal eccentricity of the pin, $d$. Besides this, the component of $\vec{d}$ that is perpendicular to the plane of the cart movement is always zero and, the others horizontal and vertical components can be calculated from the angular displacement $\alpha$ of the motor.

Assuming that there is no friction between the pin and the slot machined on an acrylic plate, the vector $\vec{f}$ only has a horizontal component, $f$ (the horizontal force that the DC motor exerts in the cart). Thus, $\vec{d}$ and $\vec{f}$ are written as

$$
\vec{d}(t)=\left[\begin{array}{c}
d \cos \alpha(t) \\
d \sin \alpha(t) \\
0
\end{array}\right], \quad \vec{f}(t)=\left[\begin{array}{c}
f(t) \\
0 \\
0
\end{array}\right] .
$$

Substituting (3) in (2), the available torque to the coupled mechanical system, $\vec{\tau}$, is

$$
\tau(t)=-f(t) d \sin \alpha(t) .
$$

Due to constraints, the cart is not allowed to move in the vertical direction. Due to the problem geometry, the horizontal motion of the cart $x$ and the angular displacement $\alpha$ of the motor are related by the constraint

$$
x(t)=d \cos \alpha(t) .
$$

Since the cart is modeled as a particle of mass $m$, it satisfies the equation

$$
m x^{\prime \prime}(t)=f(t)-k_{1} x^{\prime}(t)-c_{1} x(t) .
$$

Substituting (4), (5) and (6) in (1) one obtains the following system.

$$
\begin{gathered}
l c^{\prime}(t)+k_{e} \alpha^{\prime}(t)+r c(t)=\nu+\chi \sin \left(\omega_{1} t\right), \\
\left(d^{2} \sin (\alpha(t))^{2} k_{1}+b_{m}\right) \alpha^{\prime}(t)+\left(d^{2} m \sin (\alpha(t))^{2}+j_{m}\right) \alpha^{\prime \prime}(t) \\
+d^{2} m \cos (\alpha(t)) \sin (\alpha(t)) \alpha^{\prime}(t)^{2}-c_{1} d^{2} \cos (\alpha(t)) \sin (\alpha(t))-c(t) k_{t}=0 .
\end{gathered}
$$

\section{Dimensionless Formulation}

By taking $\alpha^{\prime}(t)=u(t)$, the system (7) the system can be rewritten as

$$
\begin{aligned}
\alpha^{\prime}(t)= & u(t) \\
c^{\prime}(t)= & \frac{\chi \sin \left(\omega_{1} t\right)-k_{e} u(t)-r c(t)+\nu}{l}, \\
u^{\prime}(t)= & -\left(u(t)\left(d^{2} \sin ^{2}(\alpha(t)) k_{1}+b_{m}\right)+d^{2} m u(t)^{2} \cos (\alpha(t)) \sin (\alpha(t))\right. \\
& \left.-c_{1} d^{2} \cos (\alpha(t)) \sin (\alpha(t))-c(t) k_{t}\right) /\left(d^{2} m \sin ^{2}(\alpha(t))+j_{m}\right)
\end{aligned}
$$

Let us write (8) in dimensionless form. Consider the following dimensionless parameters and functions given by

$$
\begin{array}{r}
t=\frac{l}{r} s, \alpha\left(\frac{l s}{r}\right)=p(s), u\left(\frac{l s}{r}\right)=\frac{r q(s)}{l}, c\left(\frac{l s}{r}\right)=\frac{k_{e} w(s)}{l}, \\
\omega_{2}=\frac{\omega_{1} l}{r}, v_{0}=\frac{\nu l}{k_{e} r}, v_{1}=\frac{d^{2} m}{j_{m}}, v_{2}=\frac{k_{e} l k_{t}}{j_{m} r^{2}}, v_{3}=\frac{b_{m} l}{j_{m} r}, v_{4}=\frac{\chi l}{k_{e} r}, v_{6}=\frac{l k_{1}}{m r}, v_{7}=\frac{c_{1} l^{2}}{m r^{2}} .
\end{array}
$$


Let us assume the following Ansatz

$$
q(s)=\omega_{0}+\epsilon z(s), w(s)=k_{0}+\epsilon w_{1}(s)
$$

where

$$
k_{0}=\frac{v_{0} v_{3}}{v_{3}+v_{2}}, \omega_{0}=\frac{v_{0} v_{2}}{v_{3}+v_{2}}
$$

Moreover, assume that

$$
v_{1}=\epsilon, v_{4}=\epsilon v_{5} .
$$

Note that in this dimensionless formulation the frequency of the external non-constant harmonic excitation is given by $\omega_{2}$, see (9). Since an internal frequency has been introduced in (10) and in order to search periodic orbits, let us take for granted the simplest case of resonance between these frequencies. So, let us assume the following near $1: 1$ resonance condition given by

$$
\omega_{2}=\omega_{0}+\epsilon k,
$$

where $k$ is a detuning parameter. It is worth to remark that $\omega_{0}$ can be interpreted as being the speed of the disk under no-load condition of the motor (see equation 4 of [7]), a measurable quantity. The detuning parameter describes how the speed varies when load is applied, that is when $d$ is increased.

Now the dimensionless time-variable will be redefined to put the system in a convenient form. If one takes into account (9)-(13) into (8), substitute

$$
s=\frac{s_{1}}{\left(\omega_{0}+\epsilon k\right)}
$$

into the resultant equation. Now, to simplify the notation, in the resulting equation, $s_{1}$ is renamed $s$, so from now on $s$ has a different meaning. From that, by considering the following change of variables

$$
p(s)=s+p_{1}(s)
$$

one gets the following equations

$$
\begin{aligned}
p_{1}^{\prime}(s)= & \frac{\epsilon z(s)-\epsilon k}{\epsilon k+\omega_{0}} \\
w_{1}^{\prime}(s)= & \frac{v_{5} \sin (s)-z(s)-w_{1}(s)}{\epsilon k+\omega_{0}}, \\
z^{\prime}(s)= & -\left(v_{6}\left(\epsilon z(s)+\omega_{0}\right) \sin ^{2}\left(p_{1}(s)+s\right)\right. \\
& +\left(\left(\epsilon z(s)+\omega_{0}\right)^{2}-v_{7}\right) \cos \left(p_{1}(s)+s\right) \sin \left(p_{1}(s)+s\right) \\
& \left.+v_{3} z(s)-v_{2} w_{1}(s)\right) /\left(\left(\epsilon k+\omega_{0}\right)\left(\epsilon \sin ^{2}\left(p_{1}(s)+s\right)+1\right)\right) .
\end{aligned}
$$

The theorems of the following section will allow us to arrive at the desired results.

\section{Some Mathematical Results}

Now, we are going to state a theorem on change of variables.

Consider $\Omega_{1} \subset \mathbb{R}^{n}, \Omega_{2} \subset \mathbb{R}^{m}$ open subsets such that $0 \in \Omega_{1}$ and $0 \in \Omega_{2}$. And the following $C^{1}$ mappings:

$$
\begin{array}{r}
\mathbf{h}_{1}: \mathbb{R} \times \Omega_{1} \times \Omega_{2} \times\left(-\epsilon_{0}, \epsilon_{0}\right) \rightarrow \mathbb{R}^{n}, \quad \mathbf{h}_{2}: \mathbb{R} \times \Omega_{1} \rightarrow \mathbb{R}^{m}, \\
\mathbf{h}_{3}: \mathbb{R} \times \Omega_{1} \times \Omega_{2} \times\left(-\epsilon_{0}, \epsilon_{0}\right) \rightarrow \mathbb{R}^{m}
\end{array}
$$

where $\epsilon_{0}>0$. Now consider the system

$$
\begin{aligned}
& \mathbf{p}^{\prime}=\epsilon \mathbf{h}_{1}(t, \mathbf{p}, \mathbf{x}, \epsilon), \\
& \mathbf{x}^{\prime}=A \mathbf{x}+\mathbf{h}_{2}(t, \mathbf{p})+\epsilon \mathbf{h}_{3}(t, \mathbf{p}, \mathbf{x}, \epsilon) .
\end{aligned}
$$


Theorem 4.1. Let $A: \mathbb{R}^{m} \rightarrow \mathbb{R}^{m}$ be a linear mapping such that $e^{T A}-I$ is nonsingular. Consider the system (18)-(19) where $\mathbf{h}_{1}, \mathbf{h}_{2}, \mathbf{h}_{3}$ are $T$ periodic mappings in the variable $t$ and $\mathbf{h}_{2}$ is $C^{2}$ mapping. Then there is a $C^{2}$ mapping $\mathbf{F}: \mathbb{R} \times \Omega_{1} \rightarrow \mathbb{R}^{m}, T$ periodic in the variable $t$, such that if one takes the change of variables

$$
\mathbf{p}=\mathbf{p}, \quad \mathbf{x}=\mathbf{y}+\mathbf{F}(t, \mathbf{p})
$$

the system (18)-(19) can be rewritten as

$$
\begin{aligned}
& \mathbf{p}^{\prime}=\epsilon \overline{\mathbf{h}}_{1}(t, \mathbf{p}, \mathbf{y}, \epsilon), \\
& \mathbf{y}^{\prime}=A \mathbf{x}+\epsilon \overline{\mathbf{h}}_{3}(t, \mathbf{p}, \mathbf{y}, \epsilon),
\end{aligned}
$$

where $\overline{\mathbf{h}}_{1}, \overline{\mathbf{h}}_{3}$ are adequate $C^{1}$ mappings, $T$ periodic in the variable $t$.

For convenience of the reader, we state the following result given in [3], page 263, Lemma 1.4 (first part) or [4], page 38, Theorem 6.1.

Theorem 4.2. Consider the following system

$$
\begin{aligned}
& \mathbf{p}^{\prime}=\epsilon \mathbf{u}_{1}(t, \mathbf{p}, \mathbf{y}, \epsilon), \\
& \mathbf{y}^{\prime}=A \mathbf{y}+\epsilon \mathbf{u}_{2}(t, \mathbf{p}, \mathbf{y}, \epsilon),
\end{aligned}
$$

where $\mathbf{u}_{1}, \mathbf{u}_{2}$ satisfy the same hypothesis that $\mathbf{h}_{1}$ of Theorem 4.1 and $e^{T A}-I$ is nonsingular. Then, given $\beta>0$ there is $\epsilon_{0}>0$ such that for all $|a| \leqslant \beta$ with $a \in \mathbb{R}^{n}$ and $|\epsilon| \leqslant \epsilon_{0}$ the system

$$
\begin{aligned}
& \mathbf{p}^{\prime}=\epsilon\left(\mathbf{u}_{1}(t, \mathbf{p}, \mathbf{y}, \epsilon)-\frac{1}{T} \int_{0}^{T} \mathbf{u}_{1}(s, \mathbf{p}(s), \mathbf{y}(s), \epsilon) d s\right), \\
& \mathbf{y}^{\prime}=A \mathbf{y}+\epsilon \mathbf{u}_{2}(t, \mathbf{p}, \mathbf{y}, \epsilon)
\end{aligned}
$$

has a unique $T$ periodic solution $(\mathbf{p}(s, a, \epsilon), \mathbf{y}(s, a, \epsilon))$ such that

$$
\mathbf{p}(s, a, 0)=a, \quad \mathbf{y}(s, a, 0)=0 .
$$

The next result gives "partial stability" of periodic solutions of the system (23)-(24) under a mild hypothesis on $A$. To the best of the authors' knowledge this result is a new one and has interest by itself.

Theorem 4.3. In (23)-(24) assume that following conditions hold

a) The mappings $\mathbf{u}_{1}, \mathbf{u}_{2}$ are $T$-periodic in the variable $t$.

b) The linear mapping $A: \mathbb{R}^{m} \rightarrow \mathbb{R}^{m}$ has all its eigenvalues with negative real part.

c) The system (23)-(24) has a T-periodic solution solution $\left(\mathbf{p}_{0}(t, \epsilon), \mathbf{y}_{0}(t, \epsilon)\right)$ for all $\epsilon \ll 1$.

Then there are $C>0, \gamma>0$, an open subset $\Omega_{3}$ of $\mathbb{R}^{m}$ with $0 \in \Omega_{3}$ and a bounded mapping

$$
K: \Omega_{3} \times(-\bar{\epsilon}, \bar{\epsilon}) \rightarrow \mathbb{R}^{n},
$$

such that the solution $(\mathbf{p}(t, \mathbf{b}, \epsilon), \mathbf{y}(t, \mathbf{b}, \epsilon))$ of (23)-(24) with initial value

$$
(\mathbf{p}(0, \mathbf{b}, \epsilon), \mathbf{y}(0, \mathbf{b}, \epsilon))=\left(\mathbf{p}_{0}(0, \epsilon)+\epsilon K(\mathbf{b}, \epsilon), \mathbf{y}_{0}(0, \epsilon)+\mathbf{b}\right)
$$

satisfies the inequalities

$$
\left|\mathbf{p}(t, \mathbf{b}, \epsilon)-\mathbf{p}_{0}(t, \epsilon)\right| \leq C e^{-\gamma t}, \quad\left|\mathbf{y}(t, \mathbf{b}, \epsilon)-\mathbf{y}_{0}(t, \epsilon)\right| \leq C e^{-\gamma t}
$$

for all $t \geqslant 0, \mathbf{b} \in \Omega_{3}$ and $\epsilon \in(-\bar{\epsilon}, \bar{\epsilon})$. 


\section{Asymptotic stability of the electromechanical system}

The system (16) can be written in the form given by (18)-(19). Now, by using the change of variables given by $(20)$, where $\mathbf{p}=p_{1}, \mathbf{x}=\left(w_{1}, z\right)$ and $\mathbf{y}=\left(w_{2}, z_{1}\right)$, one obtains

$$
\begin{aligned}
& p_{1}^{\prime}(s)=\epsilon\left(\Theta\left(s, p_{1}(s), w_{2}(s), z_{1}(s), \epsilon\right)-k\right) /\left(\epsilon k+\omega_{0}\right), \\
& w_{2}^{\prime}(s)=\frac{-z_{1}(s)-w_{2}(s)}{\omega_{0}}+\epsilon U\left(s, p_{1}(s), w_{2}(s), z_{1}(s), k, \epsilon\right), \\
& z_{1}^{\prime}(s)=\frac{v_{2} w_{2}(s)-v_{3} z_{1}(s)}{\omega_{0}}+\epsilon V\left(s, p_{1}(s), w_{2}(s), z_{1}(s), k, \epsilon\right)
\end{aligned}
$$

where

$$
\begin{aligned}
\Theta\left(s, p_{1}, w_{2}, z_{1}, \epsilon\right)= & \left(B_{41} \sin \left(2 p_{1}\right)+B_{42} \cos \left(2 p_{1}\right)\right) \sin (2 s) \\
& +\left(B_{31} \sin \left(2 p_{1}\right)+B_{32} \cos \left(2 p_{1}\right)\right) \cos (2 s)+B_{2} \sin (s)+B_{1} \cos (s)+B_{0}+z_{1}
\end{aligned}
$$

and $U, V$ are very complicated functions and there is not enough space here to write them.

Note that all components of (28)-(30) depend on $\epsilon, k$. By using Theorem 4.2 and the Implicit Function Theorem one obtains there is a $C^{1}$-mapping, $k:\left(-\epsilon_{1}, \epsilon_{1}\right) \rightarrow \mathbb{R}$, such that if $k=k(\epsilon)$ in (28)-(30) for each $\epsilon \in\left(-\epsilon_{1}, \epsilon_{1}\right)$ there exists a $2 \pi$-periodic orbit. Of course each periodic orbit depends on $\epsilon$. From Theorem 4.3 one concludes that each periodic orbit is asymptotically stable in the variables $w_{2}, z_{1}$. Note that $w_{2}, z_{1}$ stand for current and angular speed, respectively. From the physical viewpoint, in this mechanical problem, these variables are the most important. The "angle" $p_{1}$ has only secondary importance. Using Regular Perturbation Theory and after a long, but straightforward, computation one gets the form of the detuning parameter defined by 14 as a function of the system properties:

$$
\begin{aligned}
k(\epsilon)= & -\frac{\omega_{0} v_{6}}{2 v_{3}+2 v_{2}}-\epsilon\left(4 \omega_{0}^{2} v_{3} v_{7}^{2}+v_{3} v_{7}^{2}+v_{2} v_{7}^{2}-8 \omega_{0}^{4} v_{3} v_{7}-2 \omega_{0}^{2} v_{3} v_{7}\right. \\
& -2 \omega_{0}^{2} v_{2} v_{7}+4 \omega_{0}^{4} v_{3} v_{6}^{2}+\omega_{0}^{2} v_{3} v_{6}^{2}+\omega_{0}^{2} v_{2} v_{6}^{2}-12 \omega_{0}^{4} v_{3}^{2} v_{6} \\
& -3 \omega_{0}^{2} v_{3}^{2} v_{6}-6 \omega_{0}^{2} v_{2} v_{3} v_{6}-3 \omega_{0}^{2} v_{2}^{2} v_{6}+24 \omega_{0}^{4} v_{2} v_{6}-48 \omega_{0}^{6} v_{6} \\
& \left.-12 \omega_{0}^{4} v_{6}+4 \omega_{0}^{6} v_{3}+\omega_{0}^{4} v_{3}+\omega_{0}^{4} v_{2}\right) /\left(8 \omega_{0}\left(v_{3}+v_{2}\right)\right. \\
& \left.\cdot\left(4 \omega_{0}^{2} v_{3}^{2}+v_{3}^{2}+2 v_{2} v_{3}+v_{2}^{2}-8 \omega_{0}^{2} v_{2}+16 \omega_{0}^{4}+4 \omega_{0}^{2}\right)\right)+O\left(\epsilon^{2}\right) .
\end{aligned}
$$

Observe that the constant term in the expansion is due to the viscous dissipation. If there is no viscous dissipation there is no constant term. To show a periodic orbit and some other orbits being attracted to it an example is given in Figure 3. The initial conditions can be obtained by using Regular Perturbation Theory again. But their algebraic formulae are really huge ones and it is not worth to write them here.

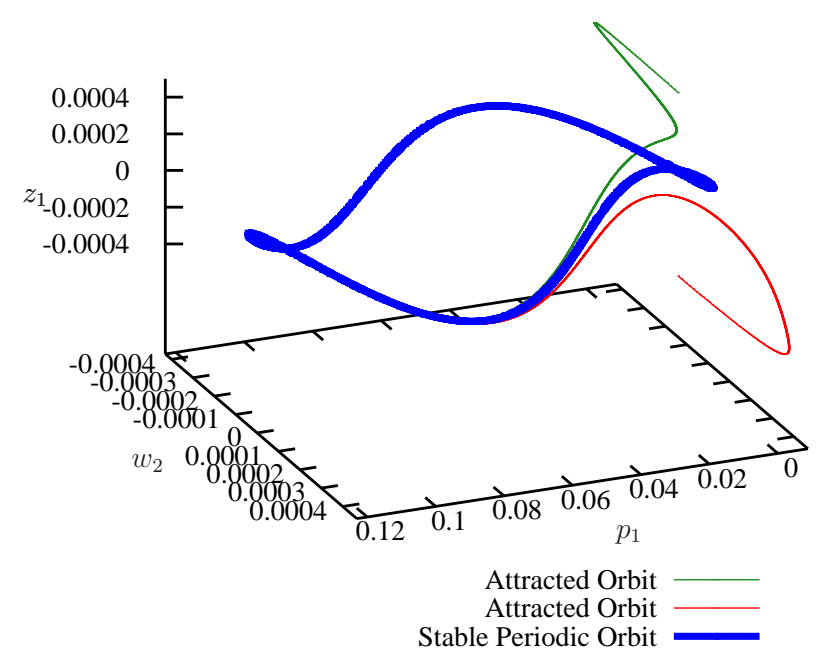

Figure 3: A periodic orbit and two attracted ones 


\section{Conclusions}

In this paper results on existence and asymptotic stability of periodic orbits of a class of electromechanical systems have been rigorously obtained by using a general approach. Here, asymptotic stability means asymptotic stability in relation to current and angular speed.

The general results, given in Section 4, afford to deal with autonomous and non-autonomous electromechanical systems in a unified way. The next step will be to investigate systems with more degrees of freedom in this area.

\section{Acknowledgments}

The first author acknowledges the support given by FAPEMIG and the second and third authors acknowledge the support given by FAPERJ, CNPq and CAPES.

\section{References}

[1] M.J. H. Dantas, R. Sampaio, R. Lima, A nonlinear electromechanical system with stable periodic orbits., in Proceedings of "12th Conference on Dynamical Systems Theory and Applications -DSTA-2013, Lodz. Dynamical Systems: Application" pp. 353-364, 2013.

[2] A. Fidlin, "Nonlinear Oscillations in Mechanical Engineering", Springer, 2006.

[3] J. Hale, "Ordinary Differential Equations", Dover Publications, Mineola, New York, 2009.

[4] J. Hale, "Oscillations in Nonlinear Systems', Dover Publications, Mineola, New York, 1992.

[5] D. C. Karnopp, D. L. Margolis, R. C. Rosenberg, "System Dynamics: Modeling and Simulation of Mechatronic Systems", John Wiley and Sons, New-York, 2006.

[6] V. O. Kononenko, "Vibrating Systems with a Limited Power Supply", London Iliffe Books LTD, England, 1969.

[7] R. Lima, R. Sampaio, Analysis of an Electromechanical Coupled System with Embarked Mass, Mecânica Computacional,, XXXI (2012) 2709-2733.

[8] A. H. Nayfeh, D.T. Mook, "Nonlinear Oscillations", John Wiley and Sons, England, 1979.

[9] Y. Rocard, "Dynamique Générale des Vibrations", Masson et Cie., Éditeurs, Paris, France, 1943. 